\title{
Roland Goigoux
}

a) roland.goigoux@univ-bpclermont.fr

\section{APPRENTISSAGE DE LA LECTURE: PROPOSITIONS DE DÉMARCHES ET D'OUTILS ADAPTÉS}

Roland Goigoux, professeur à l'université Blaise-Pascal Clermont II, spécialiste dans l'apprentissage de la lecture, a piloté l'étude « Lire et Écrire », co-financée par l'Institut français de l'éducation (IFE) et la Direction générale de l'enseignement scolaire (DGES). Cette étude a été lancée entre 2013 et 2015, et a été menée dans 131 classes concernant ainsi 2507 élèves. 3000 heures d'enseignement de la lecture et de l'écriture ont été décortiquées par une équipe de 140 enquêteurs. Cette étude de grande envergure révèle que le secret de l'efficacité est à chercher dans l'expertise des activités proposées aux élèves de CP, et notamment celles qui permettent aux élèves de «comprendre » un texte.

Cette recherche permet de faire un état des lieux des connaissances disponibles, celles que doivent mobiliser enseignants et formateurs au bénéfice de la réussite de tous les élèves. À partir de cette étude, quels sont les savoirs et savoir-faire à transmettre aux jeunes enseignants? Quels sont les impacts possibles sur la formation continue?

Dans le cadre des Grands Réseaux de Recherche de Haute-Normandie, Fabrice Hauzay, et Jean-Louis Cléro, tous deux formateurs de I'ESPE de l'Académie de Rouen, ont eu l'occasion de rencontrer Roland Goigoux. Cet échange a décliné les enjeux de la recherche menée sur la lecture et l'écriture. Des questionnements sont apparus au fil de la discussion, des réponses sont proposées avec des points d'appui qui interrogent l'efficacité pédagogique des professeurs, des méthodes, des manières de faire en classe. 


\section{Jean-Louis Cléro/Fabrice Hauzay : Dans le dossier IFE, soulevez-vous l'impact possible de la recherche sur la formation continue?}

Roland Goigoux: Pour prendre l'exemple de notre recherche (IFE), vous avez compris qu'il s'agissait de produire des connaissances nouvelles mais nous n'avions pas d'objectifs de fabrication d'outils, de conception de dispositifs de formation. On s'est bien gardé, dans la rédaction du rapport, de rédiger des éléments qui seraient formulés en termes de recommandations ou de préconisations. Mais aujourd'hui, nous sommes encore en train d'exploiter nos données. Vous imaginez bien qu'avec une telle enquête, on a un matériau dont on a actuellement communiqué un tiers de ce qu'on a recueilli. On est en ce moment en train de travailler sur des analyses de vidéos, ce qui correspond à 3000 heures de prise de vues. Quand on traite des questions dont on a pu repérer que quantitativement, elles étaient pertinentes et qu'elles avaient des effets sur les élèves, on peut maintenant descendre sur un grain plus fin. On visionne alors des vidéos en sachant lesquelles disséquer en priorité. On a donc un matériau énorme, qu'on analyse aujourd'hui pour essayer de fabriquer une sorte de typologie des manières de faire des enseignants. Cependant, aucune de ces typologies n'est directement reliée à l'impact en termes d'efficacité. Elles permettent de renvoyer au métier une image de ses propres manières de faire. On peut dire qu'il y a 5 ou 6 grandes manières de faire un peu typiques autour desquelles s'organise une diversité. Pour moi, c'est devenu un scénario de formation continue.

Donc cette activité de transposition, chacun d'entre nous, enseignant-chercheurformateur fait le travail de transposition qui consiste à dire quel matériau nous avons du côté de la recherche et dans quelle mesure peut-il nous aider à penser une ingénierie de formation. La recherche va nous permettre de proposer une description, relativement synthétique de l'action avec des manières d'agir plus ou moins typiques, emblématiques et l'ingénierie de formation que je propose à partir de là, c'est un vrai outil de formation continue.

\section{$J \mathrm{LC} / \mathrm{FH}$ : Pour que les enseignants prennent un peu de recul sur leurs propres pratiques?}

RG : À partir de ces cas concrets visionnés, la première consigne est : " et vous, à quoi ressemblez-vous ? Vous sentez-vous plus proche de l'un ou de l'autre ? ». Des enseignants réalisent qu'ils font tel geste professionnel et ils corrigent sur tel ou tel point. Cela génère une véritable réflexion qui est importante pour la formation car l'enseignant imagine les différentes alternatives à sa manière de faire. C'est le premier effet formateur, ouvrir le champ des possibles. Le deuxième 
effet formateur, c'est qu'à chaque fois, on travaille selon une méthodologie qu'on pourrait schématiser par un dessin. J'ai toujours une colonne qui est de l'ordre du gain et une colonne de l'ordre de la perte. Une colonne du côté des avantages, l'autre du côté des inconvénients. À chaque fois, on essaye de documenter les forces et les faiblesses, les intérêts et les inconvénients et les bonnes raisons qu'on a de faire un geste. Ces bonnes raisons sont souvent les avantages que le maître, qui utilise une technique, lui attribue. Mais lorsqu'il y a une alternative, on cherche ce que celle-ci peut produire comme avantages et évidemment ça nous amène à regarder les inconvénients, les limites. C'est véritablement làdessus qu'on enclenche les dynamiques formatives car, encore une fois, on ouvre la palette des possibles mais on donne des critères qui permettent de définir ce qui est important. On est toujours en train de mettre en rapport des techniques et un objectif qu'on se donne. On voit qu'avant de comparer des techniques en disant que celle-ci est meilleure que l'autre, en réalité, elles sont adéquates ou inadéquates à un but que l'on se donne. Si on est d'accord sur le but, alors on peut discuter sur l'adéquation des techniques mais on s'aperçoit que très souvent, le désaccord n'est pas sur la technique, il est sur le but ! Dans ce cas-là, c'est un autre sujet de discussion qu'il faut nourrir. Est-ce que ce but est pertinent ou pas à cet âge-là, à ce moment de l'année, sur cette notion, sur ce texte et sur ce support?

Ce matériau, que la recherche nous permet de bien théoriser, ne donne pas la réponse sur ce qu'il faut faire mais en revanche, il donne bien la trame d'une ingénierie de formation qui elle, rend les personnes plus solides dans leurs capacités de réflexion et de choix plus éclairés.

JLC/FH : Dans la synthèse du dossier, vous ne donnez pas véritablement des outils, mais vous fournissez des pistes de travail et de réflexion concrètes comme pour l'approche de la phonologie par exemple.

RG : Cet exemple-là fait partie des résultats forts de cette étude. Il y en a d'autres mais celui-là est bien identifié.

\section{JLC/FH : En prenant les données brutes, très peu commentées mais rendues le plus explicites possibles, vous en avez tout de suite tiré un enseignement.}

RG : C'était notre première idée, c'est-à-dire livrer les résultats bruts et de voir comment chacun les lisait et s'en saisissait, exactement ce que vous venez de me dire. Mais on se dit que maintenant on a laissé le temps aux gens de digérer. On nous dit partout que « ça ne suffit pas, aidez-nous à aller plus loin. Aidez-nous à vérifier qu'on a bien compris ce que vous aviez en tête lorsque vous écriviez ce que vous écrivez. D’autre part, dites-nous comment vous, vous en tireriez des 
enseignements ? » On est d'accord qu'on redevient comme vous, des formateurs parmi d'autres, peut-être un peu mieux documentés ou plus éclairés, mais des formateurs comme d'autres. II y a un moment où il faut transposer les données empiriques en actions et cette transposition, c'est toujours une prise de risque. On ne peut pas dire : " la science a montré que... Donc c'est comme ça qu'il faut faire ». II y a des moments où cette transposition, elle est elle-même interprétation. Elle est prise de risque, sur la base des données de la recherche, sur la base de vos savoirs et de vos expériences de maître, de formateur, sur les autres recherches. Ce sont des faits, ce sont des savoirs, des savoir-faire et on combine tout ça, on prend sa responsabilité de formateur et on dit: « voilà, en tant que formateur, informé comme je le suis, respectueux des résultats, je prends le risque de faire cette recommandation ». Ce sont des arguments qui permettent de soutenir des recommandations mais aucun d'entre eux n'épuisent la complexité du réel!

\section{$J \mathrm{LC} / \mathrm{FH}$ : C'est ce qui rend notre métier passionnant dans le sens où l'on teste constamment...}

RG : Vous voyez lorsqu'on dit enseignant-chercheur, le chercheur à un certain moment, s'arrête mais le formateur que je suis, veut en dire plus. J'arrête alors de parler au nom du collectif et j'assume une responsabilité individuelle qui a à voir avec ma propre expérience et mes propres connaissances car il n'est pas dit que les 45 chercheurs qui ont signé ce rapport seraient d'accord avec moi sur ma façon de tirer des enseignements sur les résultats que vous venez de rapporter. Si les maîtres, statistiquement, étudient les correspondances graphèmes-phonèmes trop lentement, ils pénalisent les élèves les plus faibles. Donc, on pourrait dire que le résultat le plus clair, c'est une mise en garde contre des maîtres qui, sous prétexte que leurs élèves sont en difficulté, adoptent une progression lente. Les mettre en garde contre cette intuition n'est certainement pas la bonne attitude.

\section{JLC/FH : C'est plutôt un avertissement, on dit aux enseignants en voulant faire le bien vous risquez de pénaliser certains de vos élèves.}

RG : Exactement! On a des résultats comme ça ! Certains d'entre eux sont lisibles en termes d'avertissement, d'autres nécessitent une élaboration plus forte. On est dans cette phase-là.

\section{$J \mathrm{LC} / \mathrm{FH}$ : Il y a également le lien entre le codage et l'encodage....}

RG : Très fort ! II faut en tirer des conséquences qui ne sont pas dans le rapport. On pourrait diminuer le temps qu'on passe à étudier l'étude du 
code sous l'angle du décodage si on investissait plus en termes de temps et en termes de tâche sous l'angle de l'encodage. On aurait probablement un bénéfice dans le rapport temps passé et apprentissages générés bien meilleur. Or, beaucoup de maîtres n'y croient pas. Et ils n'y consacrent pas le temps nécessaire.

JLC/FH : Comment expliquez-vous que certains enseignants ne croient toujours pas à ces relations entre la production d'écrits et la lecture et entre le décodage et l'encodage malgré la formation initiale et la formation continue?

RG : C'est une question compliquée. J'ai des éléments de réponses personnelles mais vous êtes d'accord que ce n'est pas cette recherche qui nous donne des éléments de réponse?

\section{$\mathrm{JLC/FH}$ : Elle illustre encore une fois différentes façons de travailler.}

RG : En effet, elle illustre. J'aurais deux ou trois grandes séries d'explications face à ce phénomène. La première chose, c'est qu'on est dans l'apprentissage de la lecture, dans la méthode de lecture et la lecture-écriture est quelque chose de beaucoup moins présent. Les parents vous demandent si leurs enfants vont bien savoir lire. Première piste, c'est le rapport entre l'enseignable et l'évaluable. On voit bien aujourd'hui l'engouement sur les techniques de fluence en lecture. Cette coqueluche qu'est devenue la lecture à haute voix. Avant, c'était horrible ! La lecture intonative du certificat de fin d'étude désuète. Et puis on baptise ça fluence, on l'américanise et ça devient la technique numéro 1 parce qu'elle fait faire des progrès significatifs, visibles, lisibles et alors vous pouvez prendre un chronomètre, une minute, tant de mots, trois semaines après vous évaluez les progrès...

La question de comment on évalue l'écriture aujourd'hui, c'est le grand parent pauvre. Si vous me dites, qu'est-ce qu'une progression normale, où doivent-ils en être sur la copie, sur la calligraphie? Sur l'orthographe, l'encodage, quelle est la norme? Qu'est-ce qu'on attend ? C'est compliqué et les maîtres de CP ont beaucoup moins les points de repère. Première famille de réponses, regardons ce rapport entre ce qui est visible et communicable.

Deuxième piste, n'a-t-on pas creusé le fossé entre des pratiques expertes de plus en plus complexes et ambitieuses, manipulables par des maîtres expérimentés mais difficilement transférables auprès d'enseignants débutants, sceptiques ou non insensibles à ça. À un moment donné, les pratiques expertes s'éloignent trop des pratiques ordinaires et le fossé à franchir est trop grand. Du coup, si on 
ne balise pas les étapes intermédiaires, celles d'une zone proximale de développement professionnel, ce modèle paraît hors de portée. On devrait être capable de dire: «Vous savez sur l'écriture, si vous faisiez simplement ça, cela changerait tellement la face des choses!»

Si on regarde la technique emblématique de la dictée à l'adulte, d'ailleurs les programmes 2015 de la maternelle mettent l'accent sur cette approche, il y a cette idée qu'il faut qu'il y ait tout le processus d'écriture. II faut qu'il y ait le projet, c'est-à-dire une intention de communiquer quelque chose d'important à un lectorat. II faut concevoir, qu'à partir de là, on doit passer des idées à la mise en mots nécessaire. Cette mise en mots, il faut être capable de la transcrire. Cette transcription va aller sur une édition qui, elle-même, ira vers le lectorat pour vérifier que l'intention de départ est satisfaite. C'est ça l'épaisseur de l'activité d'écriture. Lors du séminaire national «maternelle », mes propos sur ce sujet ont eu un fort impact pour dire « attention, vous êtes en train de tellement charger la barque que c'est décourageant ! ». Parce que ce genre de projet d'écriture, vous faites ça une fois, vous y passez trois semaines, vous en sortez exsangue et les élèves, à la fin, ont complètement oublié ce qu'était le projet de départ. On voit des classes où il s'agit de rédiger une petite histoire pour monter une pièce de théâtre de la fin du trimestre, fabriquer un ouvrage, légender la moindre photo de piscine avec un texte de trois lignes et d'autres qui disent « allez, aujourd'hui on va écrire ce mot. Comment on s'y prend? » Un mot, UN MOT, choisi par le maître dans un atelier qui est un atelier d'écriture approchée. Ce n'est pas de la dictée pure et simple mais c'est quelque chose sur une unité extrêmement réduite.

Certains vous disent « mais ce n'est pas un projet ça! ». Mais l'activité de base c'est, « vous avez une ardoise, ce mot-là on essaye de l'écrire ». « On ne sait pas m'sieur ! " Qu'est-ce qu'on sait déjà ? » et on y va ! C'est ça déjà l'écriture ou ce n'est pas ça? C'est noble ou ce n'est pas noble ? On a le droit ou on n'a pas le droit?

C'est permettre ainsi aux collègues de faire un peu plus, faire autre chose que ce qu'ils font, sans leur donner comme horizon des choses qui sont tout de suite tellement lourdes et complexes, que quand ils les ont essayées, ils n'y reviennent plus. Je pense qu'on a découragé. Ça ne veut pas dire qu'il ne faut pas donner des pratiques expertes comme horizon! Le problème c'est qu'on ne jalonne pas assez ce que pourrait être des tâches beaucoup plus modestes mais qui donneraient du jeu, en gros des techniques, des idées, des outils. Et là si on regarde la différence entre lecture et écriture, on voit qu'une des différences, c'est ma troisième explication, c'est le déficit d'outils... 
JLC/FH: Mais quand vous parlez de déficit d'outils, seraient-ce les outils donnés par les formateurs pour que les collègues aient accès plus facilement aux activités ou bien ce sont les outils que construisent les enseignants?

RG : Je parle clairement des outils qu'on propose aux enseignants comme étant des outils déjà fabriqués...

\section{JLC/FH : Nous sommes d'accord avec vous, pourtant, nous avons tous eté confrontés aux remarques « vous ne les formez pas, vous leur donnez des recettes ».}

RG : Là-dessus, ma réponse est très claire. Je suis comme vous en désaccord avec toute une partie de mon corps professionnel qui a trop longtemps raisonné comme vous venez de le dire en considérant que les outils rendaient les maîtres paresseux en leur donnant des recettes. On a des acteurs, des enseignants qui ont des connaissances, des représentations sur ce qu'est la lecture, sur ce qu'est l'écriture, sur ce qu'est apprendre à lire et à écrire et sur les techniques qui permettent d'apprendre à lire et à écrire. Et ils ont en face de ça, un certain nombre d'actions possibles, qui sont liées à des savoir-faire, voilà ! Le scénario de la formation continue et initiale classique, c'est de modifier les connaissances ou les représentations des acteurs. Une fois qu'ils sauront l'importance de l'écriture, pense-t-on, puisque ce sont des êtres rationnels, ils mettront en œuvre dans l'action les principes qui auront été mis en lumière par la formation.

Ce scénario, ce sont des dizaines d'expériences et d'années d'échecs massifs. D’où l'approche instrumentale qui est la nôtre de considérer que ce métier est un métier avec « instruments » et que, comme toutes les techniques humaines, la conceptualisation de l'action se fait par une théorisation de l'action avec un vecteur, et ce vecteur se nomme instrument et arrêtons de la caricaturer sous le terme de recette et pensons en termes de technique, pensons que les instruments sont du condensé de connaissances, de conception, de réflexion. Ces instruments vont donc être des vecteurs de cette action et de développement professionnel. C'est l'action, pensée, théorisée, grâce à l'instrument, qui a un effet de retour sur les connaissances et sur les représentations qui a l'effet formatif, qui du coup permet aussi une inventivité.

\section{JLC/FH : Ça dédramatise la démarche...}

RG: Ça permet d'oser faire car quelqu'un en qui vous avez un peu confiance vous l'a suggéré. Qui met dans la main ces outils ? Est-ce une imposition administrative ? Si demain, un gouvernement met en œuvre ce qui se prépare dans les 
cartons ministériels, c'est-à-dire l'idée qu'il y aurait des outils labellisés imposés aux professionnels dans une linéarité descendante, au nom de la science et d'un nouvel avatar du dirigisme pédagogique, alors si on a ça, je vous garantis que l'effet dont on parle ne fonctionnera pas.

Comment ces instruments sont diffusés aujourd'hui ? Par les blogs d'instits qui disent «t’as essayé le dernier truc de machin ? ». Les réseaux, c'est incroyable ! Je piste ça, c'est inouï comment ça glose, ça commente.

II y a des questions de recherche portant sur la conception d'instruments et de ce point de vue-là, si on les pense comme des instruments permettant de se développer professionnellement, alors oui, il faut toute une ingénierie de conception des outils dans ce métier comme dans les autres métiers. C'est réévaluer le concret, c'est arrêter d'avoir honte du concret comme si c'était le concret ou la théorie. La théorie qui ne sert à rien et le concret qui est utile. C'est à nous à le rehausser intellectuellement, le faire vivre et d'arrêter de faire que les enseignants chercheurs parlent de la théorie et que les maîtres formateurs parlent de la pratique. C'est ça le drame ! C'est ça qu'il faut casser !

On s'aperçoit que ce qui remobilise les enseignants, ce n'est pas un stage de management en ressources humaines, ce sont les questions au cœur du métier, quand les personnes arrivent à dépasser sur ce quoi ils se cassaient les dents.

Je trouve que souvent, la barre est mise très haut comme notamment les documents d'accompagnement des programmes de la maternelle qui n'échappent pas à cette critique.

Pour l'écriture, faisons pareil! Où sont les nœuds, où sont les leviers, quelles sont les étapes, quelles sont les tâches d'enseignement emblématiques qu'il faudrait s'assurer que les maîtres fassent suffisamment régulièrement pour que cette activité d'encodage soit finalement une activité beaucoup plus fréquente? Que cette activité soit à des moments très dirigée sous la forme d'une dictée, des ateliers de tâtonnement, ou sous des phases plus ou moins autonomes. II faudrait graduer cet univers de tâches en difficultés possibles, en autonomie ou en étayage. Si l'écriture est le parent pauvre, c'est que l'étayage est plus complexe. Le détour est généralement plus long. Les gestes professionnels ne sont alors plus inscrits dans la même temporalité. Ça demande de penser à des organisations de classe spécifiques. Je pense que cette complexité-là est trop fragmentée dans nos recherches. Vous avez les didacticiens qui s'occupent de l'activité fine de l'encodage et puis vous avez les pédagogues qui s'occupent de l'organisation du travail scolaire sauf qu'en l'occurrence là, si vous ne tenez pas tout en même temps, il y a des ingénieries qui ne fonctionnent pas. II faut pouvoir regarder comment une technique 
didactique peut avoir une vertu intrinsèque mais comment elle peut s'intégrer dans un système d'enseignement qui est plus complexe. Ce que l'on voit beaucoup dans l'étude lire et écrire c'est ça, ce sont des alchimies très complexes entre les pièces d'un puzzle énorme. Les chercheurs prennent un point et le grossissent car c'est leur objet d'étude et ils peuvent avoir raison localement et tort globalement. S'ils hypertrophient leur travail sur telles dimensions phonologiques, ça va le faire au détriment de l'écriture. Or, en réalité, le travail phonologique n'a d'intérêt et de sens que s'il est étroitement lié au travail d'écriture.

En observation de classe, on a constaté que beaucoup d'enseignants passent beaucoup de temps sur des tâches phonologiques pures, ce qui est, à mon avis, inutile. Le regain d'intérêt pour la phonologie aurait dû générer un regain d'intérêt pour l'écriture puisque la phonologie, c'est l'entrée de ce qu'on entend vers ce qu'on écrit. Sinon, on est dans une autre démarche qui est la démarche de la lecture classique, on part du graphème, on part de l'écrit et on regarde comment il se prononce. Ça c'est le chemin de la lecture. Ça veut dire que c'est une entrée graphémique. Là, les méthodes syllabiques sont cohérentes, elles sont purement graphémiques. Elles rentrent par l'écrit et elles vont vers la prononciation de l'écrit. Mais les méthodes qui disent entrer par la phonologie, devraient être des méthodes basées sur l'écriture or, elles ne le sont pas. Là, c'est une conséquence de cette recherche, c'est de dire qu'il y a une incohérence phénoménale. La montée en force de la phonologie aurait dû générer cette écriture, or, il y a une sorte de dissociation au lieu d'être le moteur. Les résultats de la recherche tels qu'ils sont rédigés ne disent pas ça! On a toutes les bonnes raisons de penser que l'encodage est bénéfique. Trouver une manière de décrire des tâches scolaires, il n'y en a pas 35, il y en a 150. II fallait donc trouver un grain qui soit fonctionnel, qui soit un grain juste du point de vue didactique mais qui soit manipulable par l'enquêteur qui en regardant le maître agir puisse ranger la tâche dans une typologie facilement manipulable « en direct ». On s'aperçoit qu'en procédant ainsi, on ne peut pas avoir une grille à 150 items. II fallait prendre la décision de qualifier ce qu'on voyait pour pouvoir faire après des affinements avec la vidéo.

Un autre résultat important de cette recherche, c'est le vrai bonus systématique pour ceux enseignant l'étude de la langue. Au CP, ce n'est pas au programme, et en même temps, c'est un peu au programme mais ce n'est pas la priorité. De quoi est-ce le signe ? Je pense que ce sont des maîtres qui ne perdent aucune occasion de faire réfléchir leurs élèves sur le sens des messages mais aussi la manière dont la langue écrite s'y prend pour transcrire. Ce ne sont pas des leçons de grammaire. Ce sont à chaque fois des pas de côté, des repérages. II y a 
des maîtres qui n'ont aucun état d'âmes à se saisir de toutes ces occasions pour mettre leurs élèves en situation de réflexion par rapport à la langue. C'est une source de jubilation de comprendre comment ça marche.

\section{JLC/FH : Dans vos ouvrages, vous vous appuyez énormément sur l'idée d'ensei- gnement explicite en lecture...}

RG : Je fais des conférences sur l'enseignement explicite. C'est une notion extrêmement galvaudée. J'ai fait une conférence en prenant 5 sens différents de cette notion-là. Certains prennent cette notion dans une vision très nord-américaine étroite dans le sens d'instruction directe. Le dossier EDUSCOL, éducation prioritaire, sur ce sujet est intéressant. Personnellement, j'ai fait une analyse du terme explicite dans les programmes, j'ai trouvé une quarantaine d'occurrences dans les programmes de cycle 3. II y a énormément de sens différents, du même mot dans ce texte. Je l'ai catégorisé en 4 blocs mais le premier sens au passage, c'est un adjectif qui ne qualifie pas l'enseignement mais l'apprentissage.

C'est également toute une réflexion sur tous ces apprentissages qui se font par la réitération de l'action, par des stabilités de l'environnement qui font qu'il y a des co-occurrences. L'organisme humain est programmé pour être capable de dégager des régularités dans un environnement relativement stable et qu'il y a plein de choses, en les fréquentant, qu'on apprend sans aucune intention de les apprendre. Ça c'est de l'apprentissage implicite et il y a beaucoup d'apprentissages à l'école qui relèvent aussi de cet apprentissage implicite.

Il y a des éléments dans les programmes qui introduisent l'idée de : à quel moment, des choses qui étaient de l'ordre de l'implicite doivent le rester et à quel moment vous les faites passer à un statut d'explicite. Elles vont être à nouveau explicitées et ils deviendront conscientisables, manipulables en tant que tel. Et si on lit bien les programmes, on voit d'ailleurs un processus qui de la maternelle au cycle 3 va vers de plus en plus d'explicite. Ce qui veut dire que ce n'est pas une modalité, c'est une préoccupation, c'est un souci et c'est quelque chose en rapport avec le développement des enfants.

Ce qui fait aujourd'hui le succès de l'enseignement explicite c'est d'abord que cela est présenté comme une réponse au défaut de démocratisation du système et une réponse aux errements des outrances des méthodes actives mal pensées, des pédagogies de projet qui, lorsqu'elles étaient excessives faisaient qu'il n'y avait pas de fréquentations suffisamment structurées, régulières, planifiées d'un certain nombre de notions. Avant de penser aux transpositions finlandaises ou nord-américaines en France, il faut quand même regarder l'adéquation de ces 
techniques à une culture professionnelle, à un rapport au métier, à la liberté pédagogique et à la ligne hiérarchique.

Je voudrais ainsi introduire une autre idée. Je suis allé regarder de près la manière dont les maîtres parlent de ce qu'ils font. Dans les outils qu'on a fabriqués, il y avait les entretiens, un petit questionnaire. On demandait aux maîtres comment ils expliquaient aux parents d'élèves la méthode qu'ils utilisaient. Et si vous mettez en relations les mots clés qu'ils livrent aux parents, les pratiques identifiées, il y a plus qu'un hiatus ! Ça veut dire qu'il y a un vrai problème de conscientisation de sa propre pratique et de la capacité à la communiquer. Par exemple, on s'aperçoit que les maîtres ont des difficultés à expliquer ce qu'ils font lorsqu'ils demandent à leurs élèves de mémoriser des mots entiers. Le petit mot « est », le petit mot « elle », le petit mot « dans », assez vite ils vont les introduire parce que s'ils veulent pouvoir faire lire des phrases, il y a un certain nombre de mots hyper fréquents qui sont souvent des mots grammaticaux indispensables et ils vont les introduire globalement. Sauf que, ils ne disposent pas de la théorie de ça ! Par exemple, que le mot est mémorisé orthographiquement c'est-à-dire comme une suite organisée de lettres. Et que si je reconnais «dans », c'est que je reconnais d-a-n-s! Ils ne savent pas l'expliquer ainsi aux parents donc ils disent, c'est global! On se rend compte qu'on a presque la moitié des enseignants de notre échantillon, qui annoncent aux parents qu'ils ont un départ global!

\section{JLC/FH : Arrivent-ils à expliquer alors pourquoi ils utilisent les prénoms des élèves comme outil ?}

RG : Vous voyez, outil pourquoi faire? II faudrait qu'ils puissent dire notamment qu'il y a deux clés dans le prénom des enfants : c'est une très belle occasion de faire de la lecture et de l'écriture, c'est une très belle occasion de dénommer les unités que sont les lettres et troisième occasion majeure, c'est d'avoir des segments qui vont être utilisés dans des processus analogiques mais ces segments qui sont des morceaux de prénoms sont notamment des syllabes. Combien de maîtres expliquent pourquoi ils s'appuient sur les prénoms! Le matériau analytique le plus fabuleux c'est évidemment la liste des prénoms parce qu'il est parmi les plus connus, les plus disponibles et évidemment dès qu'on a une Nathalie, immédiatement nous avons le NA qui va nous servir, qu'on va se mettre à entourer !

Le maître a besoin de théoriser ses gestes professionnels dans ce grain-là. On a un vrai problème de développement professionnel! C'est le fait qu'on a si peu d'occasions de travail collectif sur la didactique entre enseignants. En fait, les enseignants parlent des enfants et ils parlent de l'organisation de l'école mais ils 
ne parlent à peu près jamais de leurs techniques avec ce grain-là. Du coup, dans les moments où ils ont à communiquer, ils sont souvent très en deçà de ce qu'ils savent faire. On a des résultats qui sont une photo de ce que j'appelais un état du monde, un paysage pédagogique français et je pense que le fait de travailler les aspects descriptifs et de les modéliser, de les décrire, de les relayer et de les travailler peut-être un vrai bon levier vers la formation continue.

Enfin l'autre question est qu'avons-nous fait en maternelle pour construire des compétences qui sont aussi marquées socialement, aussi importantes sur le devenir des élèves et finalement aussi peu travaillées? Et les plus spectaculaires se situent autour de « compréhension de textes entendus » et du « vocabulaire » qui sont les plus marquées socialement, tout le monde le sait. On demande alors où on en est sur l'enseignement du vocabulaire à l'école maternelle ? II y a vraiment des pans entiers dont on sent bien qu'ils ont été trop peu investis par la recherche. Si vous faites une bibliographie sur le lexique, vous avez des tas de choses qui décrivent les carences lexicales qui génèrent des difficultés de compréhension mais si vous cherchez de l'ingénierie didactique sur le lexique de manière solide et utile pour le maître qui veut changer à peu de frais dans sa manière de faire et qui aura des effets, il y a peu de choses.

Cette année, on passe à une recherche expérimentale. On a mis dans 150 classes les scénarios construits en collaboration avec des enseignants, un album, 4 semaines de travail autour de l'album avec deux clés, apprendre à raconter et le vocabulaire. Ce qui est la clé de la production écrite, c'est la conduite monologuée et si on n'entraîne pas le contage alors il y a une partie de la pédagogie de la production écrite qui est défaillante. Cette année, on évalue ce que cela donne sur une année avec trois mois de travail puisqu'il y a trois fois un album. On a un groupe qui travaille avec nos outils qui sont des prototypes modifiés déjà deux fois. On en a qui ont les outils et l'accompagnement de conseillers pédagogiques, c'est notre deuxième groupe expérimental et on a un troisième groupe, un groupe témoin, qui a les albums et les outils d'évaluation, c'est-à-dire qu'ils savent à l'avance comment on évaluera leurs élèves à la fin. Tout le monde sait comment on évaluera les élèves à la fin et on va comparer les pratiques. Elles ne sont plus déjà des pratiques ordinaires car vous voyez, le simple fait de prévenir les maîtres que vous allez évaluer sur le fait que les élèves sachent raconter bouleverse déjà bon nombre de choses. C'est ça notre recherche actuelle pour essayer de valider les outils mais aussi la dynamique que génère cette prise en main instrumentale.

Entretien réalisé par Jean-Louis Cléro et Fabrice Hauzay

Enseignants à l'ESPE de l'Académie de Rouen. Jean-louis.clero@univ-rouen.fr Fabrice.hauzay@univ-rouen.fr 